Reprod. Nutr. Dévelop., 1988, 28 (5), 1257-1266

\title{
L'endocytose de I' " androgen-binding protein " (ABP) par les cellules principales de l'épididyme chez le rat
}

H. GÉRARD, J. L. GUÉANT ( $\left.{ }^{*}\right)$, Anne GÉRARD, Sophie FRÉMONT (*), A. El HARATE, J. P. NICOLAS $\left({ }^{*}\right), G$. GRIGNON

Laboratoire d'Histologie-Embryologie

(*) Laboratoire de Biochimie

INSERMU-308, Faculté de Médecine, B.P. 184,

54505 Vandoeuvre-les-Nancy, France.

Summary. Endocytosis of the androgen-binding-protein ( $A B P$ ) by the principal cells of rat epididymis.

The present study is based on the comparison between the radioautographic analysis of the fate of the androgen-binding protein purified from rat testes (HPLC) subsequently iodinated and injected into the epididymal lumen using a micromanipulator, and the biochemical analysis of the binding capacities of this molecule to soluble epididymal membrane extracts using HPLC and ultracentrifugation.

The various experimental conditions used here allowed to demonstrate that ABP was internalized by the epididymal epithelium and to state that this internalization was not a non specific fluid phase endocytosis but a receptor-mediated-mechanism. Indeed, from a morphological stand point, the labeled ABP was associated rather with the membranes of the endocytic apparatus than with its content. In addition, from the two lumenal cell types able to resorb seminal fluid products, only the principal cells took up the labeled ABP. Our results clearly showed that this internalization was correlated with the presence of $a^{125}$. ABP binding protein.

Since the binding of this protein molecule to ABP was saturable and Calcium and $\mathrm{pH}$ dependent, it is strongly suggested that this molecule behaves as a receptor, the ligand (or one of the ligands) of which could be ABP.

\section{Introduction.}

Chez le rat, l'androgen-binding protein (ABP) synthétisée par les cellules de Sertoli est essentiellement sécrétée dans la lumière des tubes séminifères (Gunsalus et al., 1980) et véhiculée par le fluide séminal jusqu'au canal épididymaire (Hansson et al., 1975).

Au niveau de la tête de l'épididyme son taux intraluminal diminue rapidement alors que sa concentration tissulaire devient importante (French et Ritzen, 1973; Purvis et Hansson, 1978). L'utilisation d'anticorps anti ABP permet de détecter par immunocytochimie des sites immunoréactifs notamment les corps multivésiculaires présents au pôle apical des cellules principales (Feldman et al., 1981, Pelliniemi et al., 1981). Il avait donc pu être posé comme hypothèse que l'ABP était soit dégradée, soit réabsorbée à ce niveau. 
Récemment, l'injection intraluminale d'ABP marquée à l'iode 125 combinée à la technique d'autohistoradiographie à haute résolution a permis à notre groupe de mettre en évidence, de façon directe, l'aptitude des cellules principales de la région de la tête proximale, zone 2 de Reid et Cleland (Reid et Cleland, 1957) à internaliser l'ABP du fluide séminal (Gérard et al,, 1988). De plus nous avons montré que l'ABP se lie à des extraits solubles de membranes d'épididymes $\left(K_{\mathrm{a}}=0,5 \mathrm{n} \mathrm{M}^{-1}\right)$ (Guéant et al., 1988).

II reste à déterminer s'il y a une relation de cause à effet entre la liaison de I'ABP avec la membrane plasmique des cellules épididymaires et son internalisation afin de pouvoir poser l'hypothèse d'une endocytose médiée par récepteur.

En combinant, dans différentes conditions expérimentales, l'approche morphologique, fondée sur l'autohistoradiographie à haute résolution après injection d'ABP marquée, et l'approche biochimique, fondée sur l'étude des capacités de liaison de l'ABP à des extraits solubles de membranes d'épididymes il est possible d'apporter des éléments de réponse à cette question.

\section{Matériel et méthodes.}

1) Isolement, purification, marquage par l'iode 125 et caractérisation physicochimique de l'ABP. - Ces étapes sont décrites en détail ailleurs (Guéant et al., 1986). Brièvement l'ABP est isolée à partir de testicules de rat. Elle est purifiée par chromatographie liquide haute performance (facteur de purification $\times 87500$, rendement $14 \%$ ). L'iodination selon la méthode de Markwell (1982), avec une activité spécifique de $0,1 \mathrm{mCi} / \mathrm{mg}$, ne modifie pas les principales caractéristiques physicochimiques de la molécule.

2) Microinjection de l'ABP marquée. - Les microinjections se font soit in vivo dans l'épididyme intact soit in vitro dans des fragments isolés de canal épididymaire maintenus en survie dans un milieu de culture (Hanks' 199), à l'aide d'un micromanipulateur Leitz équipé d'une micropipette de $20 \mu \mathrm{m}$ de diamètre interne. Chaque portion d'épididyme injectée reçoit environ $20 \mathrm{ng}$ d'ABP $(4 \mathrm{nCi})$ sous un volume de 0,5 à $1 \mu$ l poussé en une minute (Gérard et al., 1988).

3) Autohistoradiographie. - Les fragments d'épididyme injectés sont immédiatement fixés par immersion dans le glutaraldéhyde à $4{ }^{\circ} \mathrm{C}(2,5 \%$ dans le tampon cacodylate $0,1 \mathrm{M}, \mathrm{pH} 7,2)$ pendant $2 \mathrm{~h}$, puis postfixés par l'acide osmique (1\% dans le même tampon) et après déshydration inclus dans l'épon. Les autohistoradiographies des coupes ultrafines sont effectuées avec l'émulsion llford L4 selon la méthode du trempage. La révélation est faite par le microdol $X$ ou le phenidon après 2 mois d'exposition à $4^{\circ} \mathrm{C}$. Les coupes sont examinées à l'aide d'un microscope Siemens 102.

4) Quantification. - Les grains d'argent ont été comptés soit sur clichés photographiques, soit directement à l'écran. 
A l'écran, un cadre calibré mesurant $13,5 \times 8,7 \mu \mathrm{m}$ au grandissement $\times$ 6000 (détermination par des billes calibrées Sigma) a permis de mesurer la longueur des différents échantillons d'épithélium.

Les résultats sont exprimés par la moyenne du nombre de grains $\pm S E M$; $\mathrm{n}=8$ pour une longueur de $94,5 \mu \mathrm{m}$ d'épithélium. Les résultats sont comparés à I'aide du test de Student.

La localisation des grains d'argent associés aux corps multivésiculaires a été analysée sur photos à $\mathrm{G} \times 40000$ (grandissement initial $\times 10000, \times 12500$ ). Les demi-distances ("half distances» ou HD) entre les grains d'argent et la membrane périphérique de 200 corps multivésiculaires ont été évaluées afin de déterminer la localisation de la source émettrice par rapport à la membrane.

La valeur de $1 \mathrm{HD}$ utilisée en raison de l'épaisseur des coupes, du traceur radioactif et de l'émulsion Ilford L4 est de $900 \AA$ (Gérard et al., 1988).

5) Préparation de l'extrait de membranes épididymaires. - Après homogénéisation des fragments d'épididyme dans un tampon Tris- $\mathrm{HCl}$ contenant de l'EDTA et du glycérol, le culot de centrifugation est soumis à sonication puis mis à incuber pendant $48 \mathrm{~h}$ dans le même tampon additionné de Triton $X-100$ à $0,05 \%$. Le surnageant obtenu après $1 \mathrm{~h}$ de centrifugation à $100000 \mathrm{~g}$ à $4{ }^{\circ} \mathrm{C}$ constitue l'extrait soluble de membranes épididymaires (ESME) (Guéant et al., 1988).

6) Etude de la liaison d'ABP ${ }^{125} /$ avec les protéines de l'ESME. - La formation d'agrégats entre l'ABP iodinée et des protéines de l'extrait soluble des membranes épididymaires (ABP ${ }^{125}$ I-P-ESME) est étudiée soit par filtration sur gel sur colonne superose 6 (FPLC, Pharmacia, Suède), soit par ultracentrifugation (115000 g pendant $16 \mathrm{~h}$ ) sur gradient de sucrose (5-35\%) (Guéant et al., 1988).

Dans les 2 cas, les échantillons analysés sont préparés par incubation de $I^{\prime} A B P^{125}$ I en quantité croissante avec 0,2 ou $0,4 \mathrm{ml} \mathrm{d}$ 'ESME dans un volume total de $1 \mathrm{ml}$ de tampon Tris $\mathrm{HCl} 20 \mathrm{mM}$ pH 8,0 contenant $10 \mathrm{mM} \mathrm{CaCl}_{2}$ et $0,05 \%$ ( $\mathrm{Vol} / \mathrm{Vol})$ de Triton $\mathrm{X}-100$.

\section{Résultats.}

1) Localisation de I'ABP $125 /$ au cours des premières étapes de son endocytose par les cellules principales de la tête proximale. - Trois et cinq minutes après l'injection, les grains d'argent sont présents essentiellement au niveau de la surface apicale des cellules principales. Ils sont souvent en étroite relation avec des vésicules ou des puits recouverts (cliché 1 ) dont le rôle dans l'endocytose médiée par récepteur est bien connu (Steinman et al., 1983).

Dans les quinze minutes qui suivent, les grains d'argent se projettent successivement sur des endosomes (cliché 2), sur les corps multivésiculaires (cliché 3) puis finalement sur le compartiment Golgien.

Au niveau des corps multivésiculaires l'analyse de la distribution des grains d'argent permet d'affirmer que le traceur est fixé sur la membrane limitant ces structures (fig. 1). 

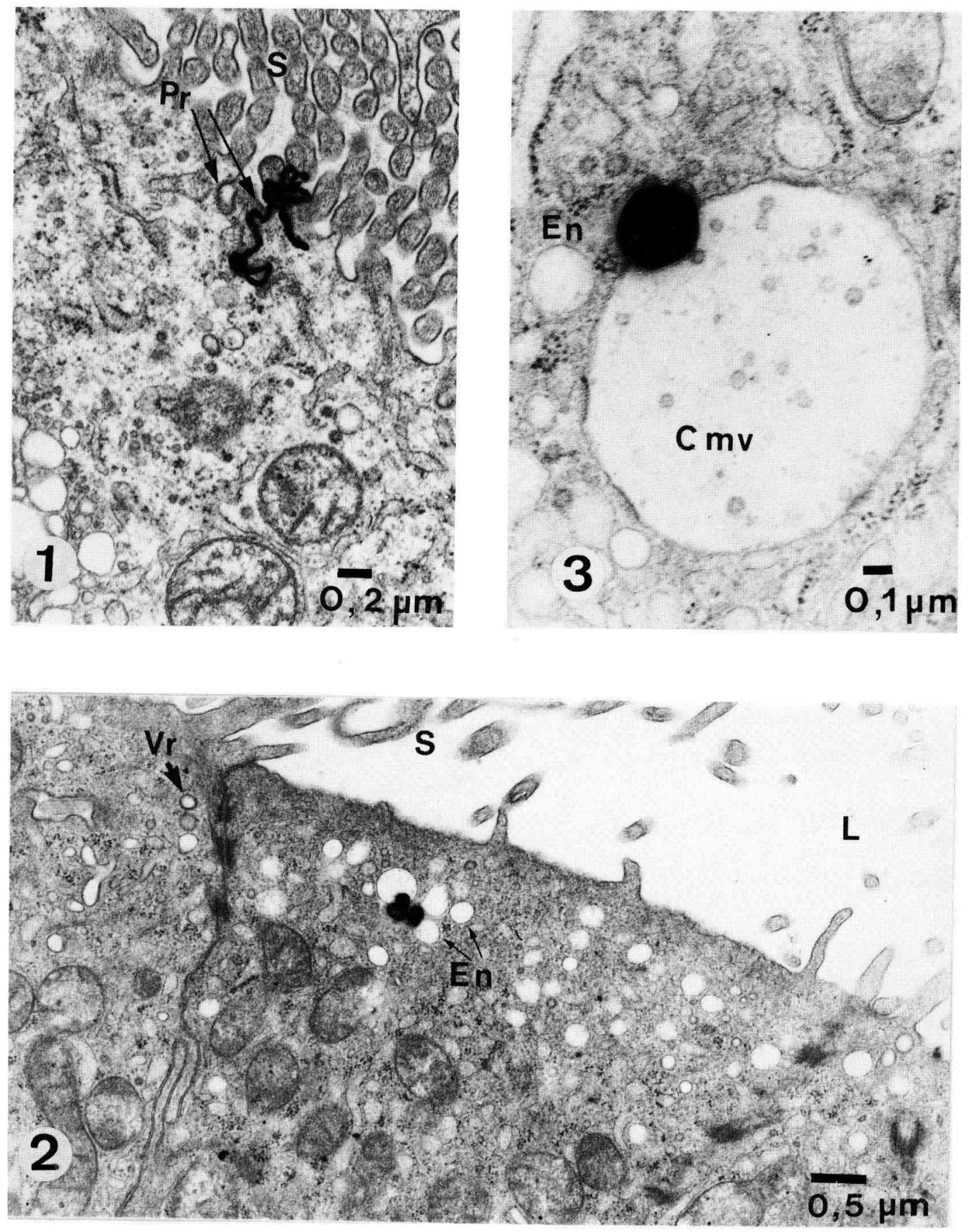


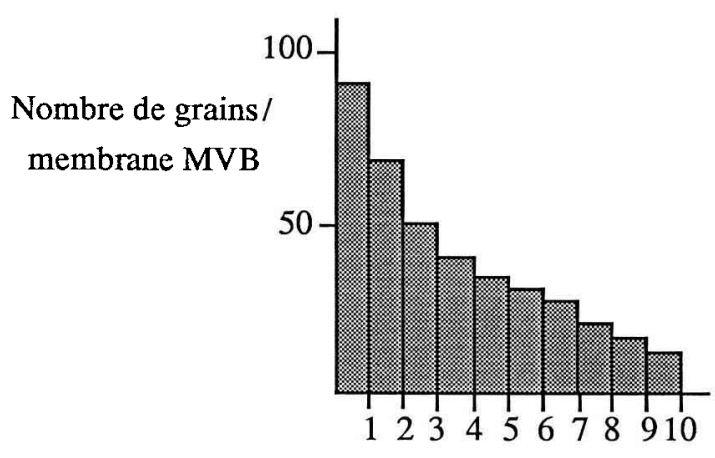

Distance $/ \mathrm{HD}$

FIG. 1. - Distribution des grains d'argent par rapport à la membrane limitant les corps multivésiculaires des cellules principales de la tête de l'épididyme. L'analyse porte sur 200 corps multivésiculaires (368 grains comptés). La distance entre le centre du grain d'argent et la membrane du corps multivésiculaire est exprimée en HD (Half-distance). Pour $\left.\right|^{125}$ I et des coupes de $800 \AA$ recouvertes d'émulsion IIford L4, 1 HD est estimé à $900 \AA$.

2) Comparaison entre l'internalisation d'ABP ${ }^{125} /$ et la quantité d'ESME liant $I^{\prime} A B P^{125}$ I dans différents territoires de l'épididyme. - Chez le rat, il est connu que la quantité d'ABP dosable au niveau du tissu épididymaire diminue progressivement de la tête vers la queue (Pelliniemi et al., 1981).

L'injection d'une même quantité $\mathrm{d}^{\prime} A B \mathrm{P}^{125}$ I dans des fragments d'épididymes isolés in vitro permet de constater qu'après 5 min d'incubation le nombre des grains d'argent présents au pôle apical des cellules épithéliales est au moins deux fois plus élevé au niveau de la tête qu'à celui de la queue (tabl. 1).

En filtration sur gel, la capacité de liaison de l'ABP marquée par les protéines de l'ESME est respectivement 10 fois et 2 fois supérieur au niveau de la tête et du corps par rapport à la queue de l'épididyme. Exprimée en terme de pmoles d'ABP ${ }^{125} \mathrm{I}$, elle est respectivement de $1,56 \mathrm{pmole} / \mathrm{mg}, 0,79 \mathrm{pmole} / \mathrm{mg}$ et de $0,16 \mathrm{pmo}-$ $\mathrm{le} / \mathrm{mg}$ de protéines au niveau de la tête, du corps et de la queue de l'épididyme.

Le complexe protéine membranaire - $\mathrm{ABP}{ }^{125} \mathrm{I}$ a une masse moléculaire estimée à $2,0 \times 10^{6} \mathrm{Da}$ en filtration sur gel (fig. 2) et est séparé en 2 pics

CLICHÉ 1. - Tête proximale de l'épididyme; cellule principale; injection intraluminale de $20 \mathrm{ng}$ de $A B P{ }^{125} \mathrm{I}(4 \mathrm{nCi}) .-3 \mathrm{~min}$ après l'injection, les grains d'argent sont fréquemment localisés à la base des stéréocils $(\mathrm{S})$, au niveau des puits recouverts $(\mathrm{Pr})$. - Exposition 2 mois à $4{ }^{\circ} \mathrm{C}$, révélation microdol $X$, Kodak.

CLICHE 2. - Queue de l'épididyme; cellule principale; injection intraluminale de $20 \mathrm{ng}$ de $\mathrm{ABP}^{125}$ | $(4 \mathrm{nCi}) .-5 \mathrm{~min}$ après l'injection les grains d'argent sont associés aux endosomes (En). Exposition 2 mois à $4{ }^{\circ} \mathrm{C}$, révélation microdol $\mathrm{X}$, Kodak.

CLICHÉ 3. - Tête de l'épididyme; cellule principale; injection intraluminale de $20 \mathrm{ng}$ de ABP ${ }^{125}$ | $(4 \mathrm{nCi}) .-10 \mathrm{~min}$ après l'injection, les corps multivésiculaires ( $\mathrm{Cmv}$ ) sont marqués. Les grains d'argent sont le plus souvent associés à la membrane périphérique de ces organites. - Exposition 2 mois à $4{ }^{\circ} \mathrm{C}$, révélation phenidon. 


\section{TABLEAU 1}

Analyse de l'endocytose d'ABP ${ }^{125}$ I dans différentes conditions expérimentales. L'activité d'endocytose est mesurée par le nombre de grains d'argent comptés dans le cytoplasme apical des cellules épithéliales 5 min après l'injection du traceur rapporté à une unité de longueur d'épithélium analysé. Le nombre de grains est exprimé par la moyenne \pm SEM établie pour des champs de $94,5 \mu \mathrm{m}$ de longueur. L'effet de chaque protocole est comparé à l'expérience contrôle (première colonne).

\begin{tabular}{|c|c|c|c|c|c|}
\hline $\begin{array}{c}\text { Localisation des grains } \\
\text { d'argent }\end{array}$ & tête & queue & tête & tête & tête \\
\hline $\begin{array}{l}\text { Protocole } \\
(n=8)\end{array}$ & $A B P{ }^{125}$ & $\mathrm{ABP}^{125}$ & $\begin{array}{c}\text { EDTA } \\
+ \\
\text { ABP }^{125}\end{array}$ & $\begin{array}{c}\text { ABP froide } \\
+ \\
\text { ABP }^{125}\end{array}$ & $\begin{array}{c}\text { Cycloheximide } \\
+ \\
\text { ABP }^{125}\end{array}$ \\
\hline
\end{tabular}
$\begin{gathered}\text { Nombre de grains d'argent/U. } 20,12 \pm 8,048,62 \pm 4,4211,81 \pm 4,822,87 \pm 0,78 \\ \text { de longueur épithélium }\end{gathered}$
$2,72 \pm 5,13$

T. test contrôle $p<0,01 \quad p<0,05 \quad p<0,001 \quad p<0,05$

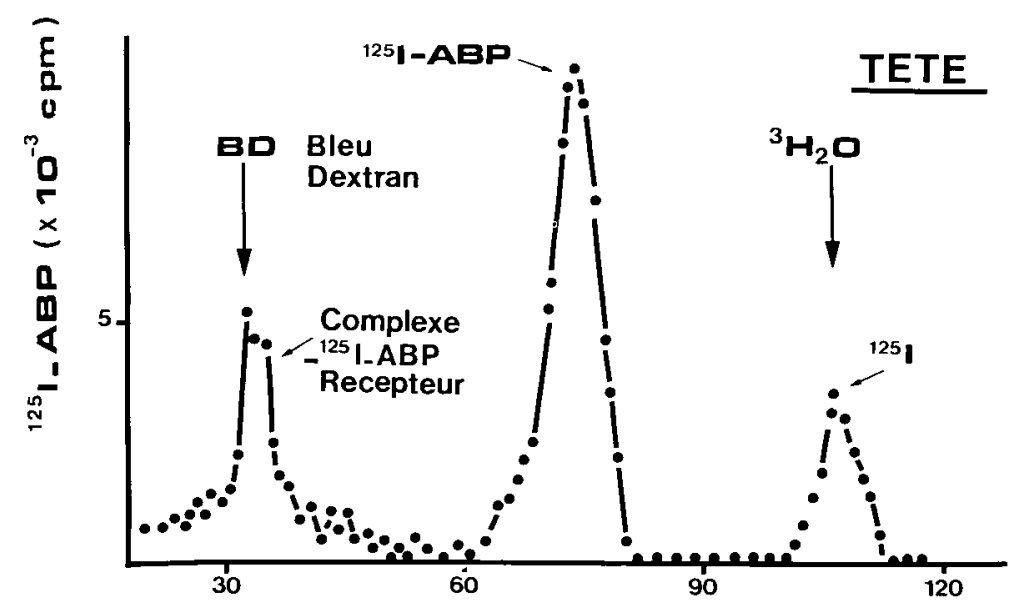

FIG. 2. - Etude en filtration sur gel de superose 6 de la liaison de l'ABP ${ }^{125} \mathrm{I}$ avec les protéines d'extrait soluble membranaire d'épididyme (ESME). L'ABP ${ }^{125}$ est incubé avec 0,2 ou $0,4 \mathrm{ml}$ d'ESME en présence de $10 \mathrm{mM} \mathrm{CaCl}$ et est élué dans une colonne FPLC $1 \times 30 \mathrm{~cm}$ à un débit de $0,5 \mathrm{ml} / \mathrm{min}$ dans du tampon tris $\mathrm{HCl} 20 \mathrm{mM} \mathrm{pH} 8,0$ contenant $0,15 \mathrm{M} \mathrm{NaCl}, 10 \% \mathrm{glycérol}$ ( $\mathrm{Vol} / \mathrm{Vol}$ ), $1 \mathrm{mM} \mathrm{CaCl}_{2}$ et $0,01 \%$ Triton $\times-100(\mathrm{Vol} / \mathrm{Vol})$

Le complexe ABP ${ }^{125}$ I protéine membranaire est élué avec du bleu de dextran (B.D.).

Le volume total de la colonne est déterminé avec de l'eau tritiée $\left(\left[{ }^{3} \mathrm{H}\right]_{2} \mathrm{O}\right)$.

correspondant à un coefficient de sédimentation respectif de $18,4 \mathrm{~S}$ et $9 \mathrm{~S}$ en ultracentrifugation sur gradient de saccharose. Les principales caractéristiques physicochimiques de la protéine membranaire sont résumées dans le tableau 2.

3) Conséquences de l'inhibition de la formation de complexes $A B P^{125}$ ESME sur l'internalisation de l'ABP ${ }^{125}$ \% - Lorsque les échantillons d'extraits 
Principales caractéristiques de la protéine membranaire épididymaire liant l'AEP marquée à l'iode.

\begin{tabular}{lc}
$\begin{array}{l}\text { Masse moléculaire apparente } \\
\text { (agrégat) }\end{array}$ & $2,0 \times 10^{6}$ daltons \\
\hline Coefficient de sédimentation & $18,45 \cdot 9,05$ \\
\hline Constante d'affinité & $0,52 \mathrm{nM}^{-1}$ \\
\hline Nombre de sites récepteurs: & $1,56 \mathrm{pmoles} / \mathrm{mg}$ protéines \\
- tête & $0,79 \mathrm{pmoles} / \mathrm{mg}$ protéines \\
- corps & $0,16 \mathrm{pmoles} / \mathrm{mg}$ protéines \\
- queue & $90 \%$ \\
\hline Inhibition de l'activité de liaison de l'ABP $(\%):$ & $87 \%$ \\
- pH 3 & $100 \%$ \\
-20 fois ABP non marquée & papaine, pronase, trypsine + chymotrypsine \\
\hline
\end{tabular}

solubles de membranes épididymaires sont incubés en présence de $10 \mathrm{mM}$ d'EDTA au lieu de $\mathrm{CaCl}_{2}$ on obtient une réduction évidente (70 à $90 \%$ ) de la quantité d'ABP ${ }^{125}$ I liée.

La préincubation de l'ESME avec de l'ABP non marquée réduit de $87 \%$ la liaison de l'ABP ${ }^{125}$.

Le chauffage de l'ESME à $60^{\circ} \mathrm{C}$ pendant 30 min ou leur prétraitement par des protéases (pronase, papaïne, trypsine et chymotrypsine) abolit la liaison de l'ABP ${ }^{125}$ I.

Enfin l'ABP ${ }^{125}$ | reste libre lorsqu'elle est incubée avec l'ESME à pH 3 (tampon citrate).

Corrélativement, des épididymes maintenus in vitro pendant $15 \mathrm{~min}$ dans un milieu sans calcium contenant $10 \mathrm{mM}$ d'EDTA et recevant une injection intraluminale d'ABP ${ }^{125}$ | diluée dans le même milieu montrent une réduction de moitié des grains d'argent présents au pôle apical des cellules épithéliales par rapport à des épididymes maintenus dans des conditions normales (tabl. 1).

De même l'injection d'un excès d'ABP non marquée (20 ng $A B P$ dans un $1 \mu l)$ juste avant l'injection d'ABP ${ }^{125} \mid\left(0,\left.2 \mathrm{ng}\right|^{\prime} A B P^{125} \mid\right.$ dans $\left.1 \mu l\right)$ réduit de plus de 5 fois le nombre des grains d'argents par rapport à l'injection première d'ABP ${ }^{125}$ I (tabl. 1).

L'administration de cycloheximide $(0,5 \mathrm{mg} / \mathrm{kg}$ - I.P.) en tant qu'inhibiteur des synthèses protéiques (Steinman et al., 1983) $2 \mathrm{~h}$ avant la microinjection $\mathrm{d}^{\prime} A B \mathrm{P}^{125}$ I diminue de près de moitié l'endocytose d'ABP (tabl. 1) ce qui suggère au moins l'intervention de protéines dans cette endocytose.

On sait que la chloroquine augmente le $\mathrm{pH}$ normalement acide des corps multivésiculaires et des lysosomes (de Duve et al., 1974). Or la préincubation des épididymes pendant $30 \mathrm{~min}$ en présence de chloroquine augmente de façon marquée le nombre des grains d'argent détectés au niveau des corps multivésiculaires. En effet, 10 min après l'injection on passe de 2,20 grains par unité de surface de corps multivésiculaires chez les témoins, à $5,60(n=4)$ et $20 \mathrm{~min}$ après on passe de 1,52 à $6,80(n=4)$. 


\section{Discussion.}

L'internalisation d'une protéine par endocytose peut s'effectuer soit par absorption non spécifique de la phase fluide, soit par adsorption sur la membrane plasmique, soit par liaison à un récepteur localisé sur cette membrane (Djakiew et al., 1984). Seule cette dernière modalité permet une internalisation à caractère spécifique et sélectif.

Nos résultats montrent que dans l'étape qui suit sa localisation au niveau des puits et des vésicules recouvertes, I'ABP est fixée sur la membrane limitant les corps multivésiculaires des cellules principales de l'épididyme. On peut donc suggérer que pendant son internalisation depuis la surface cellulaire jusqu'au compartiment des corps multivésiculaires l'ABP migre fixée sur les membranes de I'appareil endocytaire ce qui exclut une endocytose par simple absorption de la phase fluide. Ce fait confirme par conséquent les conclusions tirées de la comparaison entre l'internalisation de la peroxydase de raifort, un marqueur de l'endocytose par absorption de la phase fluide, et celle d'ABP ${ }^{125}$ I (Gérard et al., 1988). Il est également à rapprocher du caractère spécifique de l'internalisation suggéré par le fait que I'ABP ne pénètre que dans les cellules principales de l'épididyme alors que les cellules apicales, également exposées au fluide séminal, et ayant une forte activité d'absorption, restent dépourvues de marquage après injection d'ABP ${ }^{125}$ I (Gérard et al., 1988).

Nos résultats montrent de plus que les différentes quantités d'ABP détectées dans la tête et dans la queue de l'épididyme ne sont pas en relation avec la quantité d'ABP disponible au niveau du fluide séminal, mais dépendent de l'activité d'endocytose exercée par les cellules épithéliales locales. Cette activité paraît elle-même en rapport avec l'aptitude des membranes cellulaires à lier I'ABP, qui diffère selon les territoires.

Nos résultats montrent enfin que les conditions expérimentales qui limitent in vitro la liaison de l'ABP avec les extraits solubles de membranes épididymaires sont également des conditions qui réduisent la quantité d'ABP ${ }^{125}$ I détectée au pôle apical des cellules principales.

Au total, il paraît exister un parallélisme étroit entre la présence dans l'extrait soluble de membrane d'épididyme d'une molécule capable de lier I'ABP et l'activité d'internalisation de l'ABP exercée par les cellules principales.

La nature protéique de la molécule, le fait que la liaison soit calcium dépendante, $\mathrm{pH}$ dépendante et saturable, enfin les caractéristiques physicochimiques de son affinité pour l'ABP (Guéant et al., 1988) suggèrent très fortement que cette molécule fonctionne comme un récepteur (Katz et Cooper, 1974; Goldstein et al., 1979) dont I'ABP serait le ligand. Dans différents modèles d'endocytose médiée par récepteur, les corps multivésiculaires semblent constituer, par leur $\mathrm{pH}$ acide, le site privilégié de dissociation entre le récepteur et son ligand localisés sur le versant interne de leur membrane limitante (Tylo et Maxfield, 1982). Le fait que nous démontrions la localisation d'ABP ${ }^{125}$ | sur la membrane des corps multivésiculaires et son accumulation lorsqu'on augmente le $\mathrm{pH}$ de ces derniers par un traitement à la chloroquine est donc aussi en faveur de l'hypothèse récepteur-ligand. Actuellement des recherches sont en cours pour 
déterminer si d'autres ligands peuvent être en compétition avec l'ABP pour ce récepteur.

Dans l'ensemble ces résultats sont en accord avec le fait que les cellules principales de l'épididyme, notamment au niveau de la tête région 2 de Reid et Cleland, sont connues pour avoir des activités d'endocytose médiée par récepteur envers diverses substances (Djakiew et al., 1984).

Le cas de I'ABP n'est pas isolé puisqu'il semble que d'autres protéines liant les hormones stéroïdes et les transportant dans le compartiment plasmatique, soient également internalisées par les cellules des tissus cibles de ces hormones, probablement grâce à des récepteurs. C'est le cas de la CBG (corticosterone binding globulin), de la SBP (sex steroid binding protein) et de I'AFP (alpha feto protein) (Hsu et al., 1986 ; Naval et al., 1986; Hryb et al., 1985 ; Strel'chyonok et al., 1984).

A la pénétration de l'hormone stéroïde dans les cellules cibles par diffusion simple de sa forme non liée, on doit donc ajouter la possibilité d'une endocytose du complexe hormone stéroïde — protéine porteuse après interaction avec un récepteur membranaire.

Dans cette dernière éventualité la pénétration de la testostérone dans les cellules cibles de l'épithélium épididymaire ne serait plus sous la seule dépendance de la concentration de sa fraction libre dans le fluide séminal; par ailleurs lors des premières étapes de son transport intracytoplasmique I'ABP pourrait protéger la testostérone et/ou assurer sa libération au niveau de sites intracellulaires bien définis.

$5^{e}$ Congrès de la Société d'Andrologie de langue française, Paris, décembre 1987.

\section{Références}

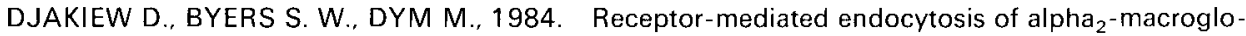
bulin and transferrin in rat caput epididymal epithelial cells in vitro. Biol. Reprod., 31 , 1073-1085.

de DUVE C., DEBARSY T., POOLE B., 1974. Lysosomotropic agents. Biochem. Pharmacol., 23. 2495.

FELDMAN M., LEA O. A., PETRUSZ P., TRES L. L., KIERZENBAUM A. L., FRENCH F. S., 1981. Androgen-binding protein. Purification from rat epididymis characterization and immunocytochemical localization. J. biol. Chem., 256, 5170-5175.

FRENCH F. S., RITZEN E. M., 1973. A high affinity androgen-binding protein (ABP) in rat testis : evidence for secretion into efferent duct fluid and absorption by epididymis. Endocrinology. 27, 437-439.

GÉRARD A., KHANFRI J., GUÉANT J. L., FRÉMONT S., NICOLAS J. P., GRIGNON G., GÉRARD H., 1988. Electron microscope radioautographic evidence of in vivo androgen-binding protein internalization in the rat epididymis principal cells. Endocrinology, 122 1297-1307.

GOLDSTEIN J. L., ANDERSON R. G. W., BROWN M. S., 1979. Coated pits, coated vesicles and receptor-mediated endocytosis. Nature, 279, 679-685.

GUÉANT J. L., KHANFRI J., GERARD H., FREMONT S., GÉRARD A., GRIGNON G., NICOLAS J. P., 1986. Purification of androgen-binding protein from rat testis using high performance liquid chromatography and physicochemical properties of the iodinated molecules. FEBS Letters, 207. 280-285. 
GUÉANT J. L., FRÉMONT S., KHANFRI J., GÉRARD A., NICOLAS J. P., GÉRARD H., $1988 . \quad$ Biochemical evidences for a receptor-mediated uptake of rat androgen-binding protein by epididymis. Steroids (sous presse).

GUNSALUS G. L., MUSTO N. A., BARDIN C. W., 1980. Bidirectional release of a Sertoli cell product, androgen-binding protein, into the blood and seminiferous tubule. In STEINBERGER A., STEINBERGER E., Testicular development, structure and function. Raven Press, New York, p. 291.

HANSSON V., RITZEN E. M., FRENCH F. S., NAYFEH S. N., 1975. Androgen transport and receptor mechanisms in testis and epididymis. In HAMILTON D. W., GREEP R. O., Handbook of physiology, section 7, vol. 5. Am. Physiol. Soc., Washington, D. C., p. 137.

HRYB D. J., KHAN M. S., ROSNER W., 1985. Testosterone-estradiol-binding globulin binds to human prostatic cell membranes. Biochem. biophys. Res. Comm., 128, 432-440.

HSU R. S., SIITERI P. K., KUHN R. W., 1986. Interactions between corticosteroid-binding globulin (CBG) and target tissues. In Binding proteins of steroid hormones. Coll. INSERM, J. Libbey Eurotext Ltd, 149, 577-591.

KATZ M., COOPER B. A., 1974. Solubilized receptor for intrinsic factor vitamin B12 complex from Guinea pig intestinal mucosa. J. clin. Invest., 54, 733-739.

MARKWELL M. A. K., 1982. A new solid state reagent to iodinate proteins. I. Conditions for the efficient labeling of antiserum. Ann. Biochem. 125, 427-432.

NAVAL J., LABORDA J., VILLACAMPA M. J., CALVO M., GEUSKENS M., URIEL J., 1986. Alpha foetoproteine (AFP) récepteurs d'alphafoetoprotéine et cancer. In Binding proteins of steroid hormones. Coll. INSERM, J. Libbey Eurotext Ltd, 149, 523-536.

PELLINIEMI L. J., DYM M., GUNSALUS G. L., MUSTO N. A., BARDIN C. W., FAWCETT D. W., 1981. Immunocytochemical localization of androgen-binding protein in the male rat reproductive tract. Endocrinology, 108, 925-931.

PURVIS K., HANSSON V., 1978. Androgens and androgen-binding protein in rat epididymis. $J$. Reprod. Fert., 52, 59-63.

REID B. L., CLELAND K. W., 1957. The structure and function of the epididymis. I. The histology of the epididymis. Aust. J. Zool., 5, 223-252.

STEINMAN R. M., MELLMAN I. S., MULLER W. A., COHN Z. A., 1983. Endocytosis and the recycling of plasma membrane. J. Cell. Biol., 96, 1-27.

STREL'CHYONOK O. A., AVVAKUMOV G. V., SURVILO L. I., 1984. A recognition system for sex hormone-binding protein-estradiol complex in human decidual endometrium plasma membranes. Biochim. Biophys. Acta, 802, 459-466.

TYLO B., MAXFIELD F. R. 1982. Rapid acidification of endocytic vesicles containing alpha macroglobulin. Cell, 28, 643-665. 